\title{
Cost Analysis of a Managed Care Decentralized Outpatient Pharmacy Anticoagulation Service
}

ROBERT J. ANDERSON, PharmD

\begin{abstract}
OBJECTIVES: To determine the per-patient-per-month (PPPM) cost of a decentralized outpatient pharmacy anticoagulation service (OPAS) in patients with chronic atrial fibrillation (AF) who were maintained on warfarin sodium therapy in a managed care setting, to compare the annual costs versus the risk for stroke, and to assess the quality of the anticoagulant management.
\end{abstract}

METHODS: Data were collected retrospectively from clinical, research, and administrative claims databases. Patient demographic data were stratified to include age and risk factors for stroke. Inclusion criteria for the study were adult patients ( $>18$ years) who were maintained on chronic warfarin therapy with a diagnosis of AF (diagnosis code 427.31) and continuously enrolled during calendar year 2000. The cost analysis included the personnel cost of clinical pharmacy specialists, direct and indirect cost of laboratory tests for international normalized ratios (INR), and anticoagulant (warfarin plus bridge therapy with a low molecular weight heparin) drug cost and dispensing fee. The percentage of INR values within or near target was used to evaluate the effectiveness of the service.

RESULTS: A total of 97 patients on chronic warfarin therapy for AF were identified for cost analysis. The demographics for these patients included the following: $71 \%$ were male, with $32 \%$ of the patients over the age of 75 years, and $60 \%$ had 1 or more identifiable risk factors for stroke. Utilizing established criteria, $80.4 \%$ of the sample was considered to be at high risk for ischemic stroke. A majority of the patients $(94.8 \%)$ had nonvalvular disease, with an INR goal in the range of 2 to 3 in $91.8 \%$ of the cases. The PPPM cost for the OPAS monitoring service was $\$ 51.25$, distributed as $\$ 13.78(27 \%)$ in personnel costs for monitoring pharmacists, $\$ 18.38$ (36\%) for lab tests, and $\$ 19.09$ (37\%) for anticoagulant drug costs. These costs did not significantly differ among patient groups with various risks for ischemic stroke. For nonvalvular AF patients, the percentage of INR values within each individual patient's specific INR goal range was $60.4 \%$; the percentage within or near goal was $74.6 \%$.

CONCLUSION: The average PPPM cost for pharmacist and laboratory monitoring as well as anticoagulant medication for CY 2000 was estimated to be $\$ 51.25$. The annual costs were comparable among AF patients with different risks for ischemic stroke. The percentage of INR values within the individual patient's stated target goal was $60.4 \%$. Effective monitoring to maintain patients within their target INR goal is relatively inexpensive compared with the cost of complications such as ischemic stroke or intracranial bleeding.

KEYWORDS: Atrial fibrillation, Anticoagulation service, Pharmacy, Cost analysis, Managed care, Per patient per month (PPPM)

J Manag Care Pharm. 2004;10(2):159-65
A trial fibrillation (AF) affects approximately 2.3 million people in the United States and is an independent risk factor for the development of stroke. The incidence of AF increases with age, with an incidence of $9 \%$ in patients older than 80 years. ${ }^{1}$ The median age of patients with a diagnosis of AF is 75 years. ${ }^{2}$ The overall rate of ischemic stroke in those not treated with antithrombotic therapy averages approximately 5\% per year, ${ }^{3}$ with an incidence of $23.5 \%$ in patients older than 80 years in the Framingham Heart Study. ${ }^{4}$

To prevent initial and recurring ischemic stroke, many highrisk patients with $\mathrm{AF}$ are maintained on an oral anticoagulant such as sodium warfarin. ${ }^{3,5}$ This medication has a narrow therapeutic-toxic index with high interindividual variability requiring frequent international normalized ratio (INR) laboratory testing to determine the patient's clotting time. For most patients with nonvalvular AF, a goal INR is in the range of 2 to 3. Insufficient doses $(\mathrm{INR}<2)$ can lead to ischemic stroke and death, ${ }^{6}$ and excessive doses (INR $>4$ ) increase the risk of intracranial bleeding. ${ }^{7}$ Those patients most likely to benefit from anticoagulant therapy (the elderly) are also those most at risk for bleeding complications. ${ }^{8,9}$ An intention-to-treat analysis of pooled data from randomized controlled trials demonstrated a relative stroke risk reduction of $68 \%$ for warfarin compared with controls. ${ }^{3}$ It has been estimated that more than $50 \%$ of strokes could be prevented if patients could be maintained within their INR goal. ${ }^{9}$ The beneficial effects of warfarin for stroke prevention in AF have been confirmed in a usual clinical care practice in a managed care population. ${ }^{10}$

To achieve beneficial risk reduction with warfarin, high-risk patients with AF must be prescribed warfarin and maintained in the therapeutic range. A study in eligible AF patients revealed that only $41 \%$ received a discharge prescription for warfarin despite no contraindications. ${ }^{11}$ For AF patients who are prescribed warfarin, the laboratory values are often within target range less than $50 \%$ of the time. ${ }^{12}$ Thus, there is a high per-

\section{Author}

ROBERT J. ANDERSON, PharmD, is a clinical pharmacy specialist, Kaiser Permanente, and a professor, Mercer University Southern School of Pharmacy, both in Atlanta, Georgia.

AUTHOR CORRESPONDENCE: Robert J. Anderson, PharmD, Professor, Mercer University Southern School of Pharmacy, 3001 Mercer University Dr., Atlanta, GA 30341. Tel: (678) 547-6382; Fax: (678) 547-6384;

E-mail: anderson_rj@merceredu

Copyright $\odot$ 2004, Academy of Managed Care Pharmacy. All rights reserved. 
centage of AF patients who continue to be at high risk for ischemic stroke even if warfarin is prescribed.

A structured or systematic anticoagulation service has been shown to improve the quality of care..$^{12}$ The management responsibilities in these clinics are often assigned to specialty nurses, nurse practitioners, physician assistants, or clinical pharmacists under a physician's direction. These providers are often responsible for patient education, including counseling on diet and drug interactions, ordering and reviewing INR laboratory values, making necessary dosage adjustments, stressing drug adherence, and documenting interaction with the patient. In addition, many such clinics are organized to interface with referral physicians on procedures or surgeries requiring the temporary discontinuation of warfarin and initiation of low molecular weight heparin (enoxaparin) to minimize bleeding risks.

What is the cost to a managed care organization for providing an anticoagulation service? Do costs differ based on risk factors for stroke? How effective is the service in terms of keeping patients within their INR target goal, thus minimizing medication-related problems and costly hospitalizations and emergency room visits?

\section{Description of Facilities and the Outpatient Pharmacy Anticoagulation Service}

Kaiser Permanente Georgia Region has more than 275,000 members at 11 decentralized medical centers in the Atlanta metropolitan area. During CY 2000, more than 1,200 patients $(0.4 \%$ of members) were prescribed warfarin therapy for several indications such as deep-vein thrombosis, pulmonary embolism, AF, myocardial infarction, congestive heart failure, genetic coagulation deficits, or cancer. These patients may be on short-term ( $<3$ to 6 months), intermittent, or lifelong therapy, depending on the risk of recurrent thromboembolic disease and stroke.

In 1995, a protocol-driven, decentralized outpatient pharmacy anticoagulation service (OPAS) was implemented, utilizing trained clinical pharmacy specialists (CPSs). With physician input and oversight, a protocol was developed and approved by the pharmacy and therapeutics committee. This protocol has been modified over the years to reflect advances and current recommendations in the medical literature. The referring physician makes the decision on initiating anticoagulant therapy and sets the INR goal for each patient.

OPAS provides coverage 24 hours a day, 7 days a week, including weekend on-call service. These trained pharmacists assume responsibility for monitoring the patient at discharge from the hospital, In calendar year 2000, there were 7 CPS pharmacists and a pharmacy resident who provided anticoagulant monitoring services from 9 different (decentralized) locations. These pharmacists were responsible for ordering the laboratory tests and adjusting the doses per protocol to achieve the desired INR goal as set by the primary care physician or specialist. Patient counseling in person or over the phone was provided. If patients were not available for follow-up lab visits by phone, letters were sent to improve compliance to the prescribed regimen.

\section{Objectives}

The primary objectives of this study were to (1) determine the per-patient-per-month (PPPM) cost of a decentralized OPAS focusing on patients with chronic AF maintained on warfarin therapy and (2) compare the costs of OPAS among patients with different risks for ischemic stroke. A secondary objective was to assess the quality of anticoagulation control, as measured by percentage of INR values within or near goal for each patient.

\section{Methodology}

Data on potential AF patients were collected retrospectively from a relational clinical database using Paradox software with data fields that included patient medical record number, start date in the anticoagulation service, indication for warfarin, and INR goal. The data that were extracted from research and clinical databases included gender, date of birth, enrollment status, diagnosis of AF (International Classification of Diseases, 9th revision, Clinical Modification, ICD-9-CM code 427.31), and comorbid risk factors for stroke, enrollment dates, warfarin usage, cost of drug, and the cost, number, and value of INR tests. A computerized review of the patient medical record was completed to verify risk factors for stroke. Ethnicity, aspirin use and dose, and smoking or alcohol history could not be reliably determined because this information was not consistently recorded in either the databases or the patient medical record.

Inclusion criteria for the study were as follows: adult patients ( $>18$ years) who were maintained on chronic warfarin sodium therapy with a diagnosis of AF (ICD-9-CM code 427.31). The diagnosis of $\mathrm{AF}$ and initiation of warfarin therapy were documented prior to CY 2000 from the clinical OPAS database. Patients with valvular disease who were at a very high risk for stroke were not excluded. Patients with paroxysmal or new onset $\mathrm{AF}$ were excluded from the analysis as were those with less than 12 months of continuous enrollment during the year 2000 .

Personnel costs include only those incurred from consultations with the chronic AF patients. All other indications for short- or long-term warfarin therapy, including paroxysmal and new onset AF (since 2000), were excluded in the analysis. In addition, OPAS was not a dedicated anticoagulation service at the time of the study, and pharmacists had other clinical responsibilities such as cholesterol management.

The chronic AF sample was then stratified by risk of ischemic stroke, utilizing guidelines established by the American College of Chest Physicians (ACCP). ${ }^{5}$ High risk was defined as an AF patient with 1 or more of the following characteristics: (1) prior stroke, transient ischemic attack, or systemic embolus; (2) aged $>75$ years; (3) a history of hypertension or poor left-ventricular systolic function; or (4) either rheumat- 
ic mitral valve disease or those with prosthetic heart valves. Patients with multiple moderate risk factors were also considered high risk. Moderate risk was defined as an AF patient with only 1 of the following characteristics: aged 65 to 75 years, diabetes mellitus, or coronary artery disease. Low risk was defined as an AF patient aged less than 65 years with no clinical or electrocardiogram evidence of cardiovascular disease. This risk stratification was utilized in both analyses of the cost and effectiveness of the anticoagulation service.

The total annual and PPPM costs for OPAS included anticoagulant drugs, INR laboratory tests, and clinical pharmacist monitoring costs for each individual patient for CY 2000. The databases provided acquisition costs for medications and INR tests. Usual and customary fees for third-party reimbursement were utilized for pharmacy dispensing and laboratory fees. The drug costs are actual annualized costs divided by 12 to derive PPPM cost. Not every patient received a continuous 30-day supply; the 97 patients averaged 6 prescriptions for warfarin per year.

The monitoring costs for the anticoagulation service were based on the total number of INR laboratory values since each test generated an automatic patient consult. The amount of personnel time required for each patient consult included reviewing the lab result and INR goal for the patient, contacting the patient (usually by telephone) to review and discuss results, counseling the patient on diet and new medications (including nonprescription drugs, diet supplements, and herbals), adjusting drug doses, writing new prescriptions, setting the next lab appointment, and documenting the intervention in the database and patient medical record. This personnel time also included contacting the physician and pharmacist about a change in drug or dose due to a potential drug-drug interaction. The time for each intervention was estimated and documented by the CPS to be in the range of 10 to 20 minutes; the time varied depending on the complexity of the case, experience of the pharmacist, and ability to contact the patient. Some patients required multiple efforts or certified letters to make successful contact for dosage adjustment and laboratory retesting. An average of 15 minutes per consult was utilized for this cost analysis.

The costs of medication and monitoring for OPAS also included patients who were candidates for "bridging" therapy during CY 2000. Bridging is defined as the need to temporarily stop warfarin therapy due to dental, outpatient, or inpatient procedures or surgeries that involve a high risk of bleeding. Patients were tapered off the warfarin and placed on self-administered subcutaneous injections of low-molecular-weight heparin (enoxaparin); after undergoing the procedure or surgery, they resumed therapy with warfarin. This process of coordinating care between the referral physician and the patient, educating the patient, developing a day-to-day drug-dose planner, overlapping therapies, adjusting the doses, writing new prescriptions, educating the patient on injection techniques, and documenting the data in the medical record was estimated at 3 hours per consult by the CPSs and was included in the cost analysis for the anticoagulation service. The personnel cost for the OPAS monitoring service was then estimated based on the time of each INR/bridging consult per patient, utilizing the average salary and benefits for a CPS for CY 2000 for a 24-hour, 7-day per-week service.

The actual monthly INR values for CY 2000 were used to assess percentages within or near goal as a surrogate marker for evaluating the quality of the anticoagulation service. Five patients with greater than 2 months between INR values were identified as potentially noncompliant and excluded from this analysis. If more than 1 INR was ordered in any 1 month, an average was calculated and compared with that patient's individual goal. The percentage of lab results near the INR goal was also determined and defined as a laboratory variation of \pm 0.2 units from the target INR. All data were collected in confidence such that no patient or provider was identified, in compliance with the research protocol approvals granted by the university and health maintenance organization institutional review boards.

\section{Statistical Analysis}

The mean and standard deviation for the annual costs for each of the components of the cost analysis were identified and compared by risk group utilizing a 1-way factorial analysis of variance (ANOVA). Pair-wise comparisons of the various costs of the risk groups were compared utilizing the Tukey test to test for significant differences in the costs among the groups. A PPPM cost for OPAS was then determined.

$P$ values were determined for the categorical variable (INR test in target range or not), utilizing a chi-square test 2-way contingency table in AF patients with different risk factors for stroke. The INR values at goal for patients with low and moderate risks for stroke were combined and compared with those for patients at high risk.

\section{Results}

A total of 97 patients with AF on chronic warfarin therapy who met the inclusion/exclusion criteria were identified for the cost analysis. Eighty-nine patients (91.8\%) had an INR goal of 2 to 3 as specified by the referring physician. Five patients (5.2\%) had higher-risk valvular AF with more aggressive INR goals of 2.5 to 3.5. The remaining 3 patients had more focused goals with INR targets in the range of 2 to 2.5 (2 patients) or 3 to 3.5 (1 patient).

This population consisted of $71 \%$ males and $29 \%$ females. The incidence of individual risk factors for ischemic stroke is displayed in Figure 1. The incidence of patients with more than 1 risk factor was $60 \%$ (Figure 2). Utilizing the risk stratification from the ACCP criteria, there were $80.4 \%$ of the AF patients at high risk of ischemic stroke, $13.4 \%$ with moderate risk, and $6.2 \%$ with low risk who were maintained on chronic warfarin therapy. 


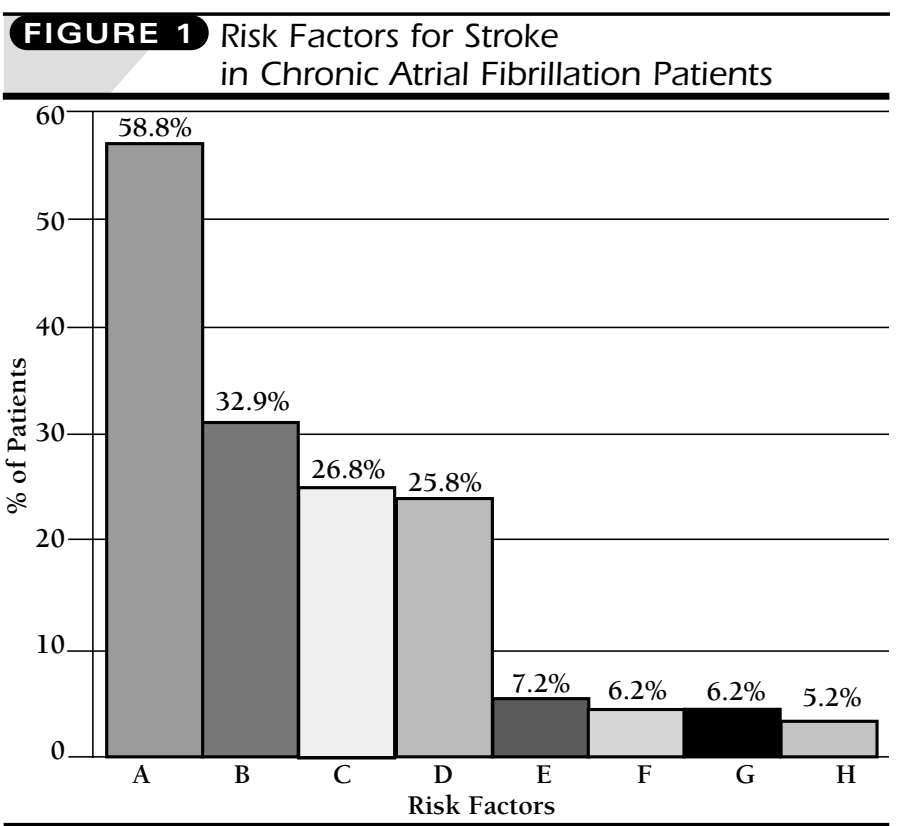

$A=$ hypertenion; $B=$ age $>75 ; C=$ diabetes mellitus; $D=$ congestive heart failure; $E=$ stroke; $F=$ transient ischemic attack; $G=$ myocardial infartion; $H=$ valve disease

\section{FIGURE 2 Percentage of Multiple Risk Factors}

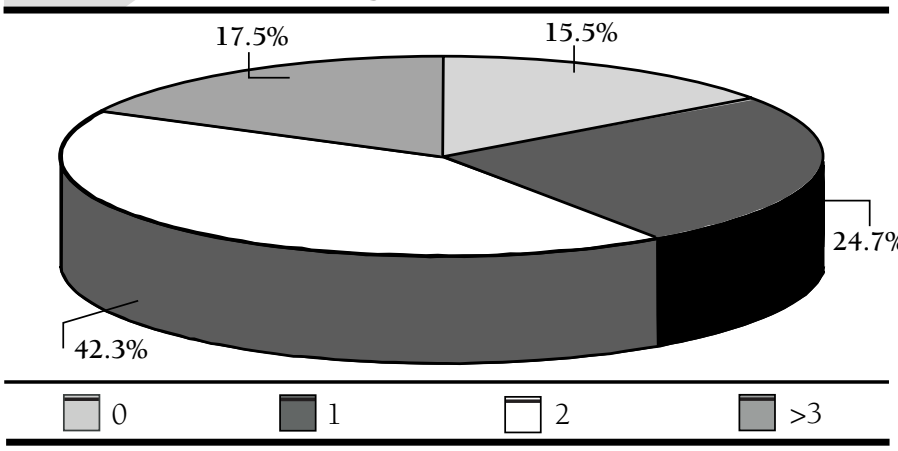

There were 593 prescriptions dispensed for warfarin during CY 2000 for the 97 patients, or an average of 6.1 prescriptions for warfarin per patient per year, or approximately 1 every other month. This result occurred for these patients on continuous warfarin therapy because many patients received a 60-day, 90-day, or even larger supply per prescription fill, in order to lower their out-of-pocket (copay) cost. Low-molecular-weight heparin (enoxaparin) bridging was prescribed for 4 patients (4.1\%) in the sample during the study. There were 1,288 INR tests completed for the 97 patients during CY 2000, or an average of 1.1 tests PPPM. Each of these INR tests generated a pharmacist-patient intervention that was estimated at 15 minutes in personnel time for each.

\section{Cost Analysis}

The mean cost for each component of OPAS for each of the AF risk groups is provided in Table 1 . There were no significant differences in the annual costs for the pharmacist monitoring service $(P=0.614)$ or laboratory costs $(P=0.62)$. The mean cost of medications in the low-risk group was higher and significantly differed from the other risk groups $(P<0.017)$. This higher cost in the lower-risk group appeared to be an anomaly due to both the small number of patients $(n=6)$ and higher medication cost in 1 patient who was prescribed the expensive enoxaparin bridging therapy. Indeed, when the outlier patient was deleted from the medication cost analysis, there was no significant difference in the ANOVA $(P=0.205)$ or among different risk groups in the Tukey post-ANOVA pair-wise comparison. The mean total annual OPAS cost also differed because of the outlier ( $P<0.03)$, but no difference $(P=0.168)$ when the outlier patient was excluded from the analysis.

A PPPM cost was then determined from the mean annual total cost. The subtotal of the cost of the medications (warfarin and enoxaparin), laboratory, and pharmacist monitoring for all chronic AF patients was determined to be an average of $\$ 51.25$ PPPM for all risk groups in CY 2000 dollars. The breakdown of costs for each component of the anticoagulation service is listed in Table 2 .

\section{Assessing the Effectiveness of the Anticoagulation Service}

There were 92 patients who met the compliance inclusion criteria for assessing the quality of warfarin control in OPAS. Five patients (or 26 INR values) were excluded because of a $\geq 2$-month time interval between INR results. There were a total of 914 monthly INR values completed on the remaining 92 patients with chronic AF, including 5 patients with valvular disease.

For the 87 nonvalvular AF patients, the percentage of INR values within each individual patient's specific goal was $60.4 \%$. In these patients, $14.3 \%$ of the lab results had an INR above 3 . With a 0.2-unit allowance for lab variation (i.e., 3.01 to3.2), the percentage of INRs above target goal was adjusted to $10.5 \%$. Only $1.6 \%$ of the INR values $(n=14)$ were greater than 4 , where the incidence of intracranial bleeding increases. The nonvalvular patients represented 1,044 months of potential exposure to complications. Thus, the potential risk exposure for bleeding secondary to excessive warfarin dosing was only $1.3 \%$ (14 months/1,044 months).

The percentage of INR lab results less than goal was $25.2 \%$; however, with allowances for lab variation (between 1.8 and 2), this percentage was lowered to $14.6 \%(n=126)$. Thus, the potential risk exposure for ischemic stroke secondary to suboptimal warfarin may be as low as $12.1 \%$ (126 months/1,044 months) in AF patients managed by OPAS. Patients were more likely to be under than over their target INR goal in OPAS. The percentage of INR values within or near goal for the nonvalvular AF patients was $74.6 \%$. 
The 5 high-risk valvular patients with more aggressive INR goals (2.5 to 3.5) were more difficult to control, with only $39.6 \%$ at goal and $56.6 \%$ near goal (i.e., a range of 2.3 to 3.7 ). One patient was not at or near goal for the entire year. With the inclusion of these AF patients with valvular disease, the percentage of time within INR goal was lowered to $59.2 \%$. With allowances for laboratory error at both ends of the range (0.2 units), the overall percentage of INR values within or near goal was $73.5 \%$ for both nonvalvular and valvular patients. There was no statistically significant difference $(P=0.546)$ in percentage of INR values at goal in patients with low and moderate versus high risk for ischemic stroke (Table 3).

\section{Discussion}

The PPPM for OPAS was estimated to be $\$ 51.25$ in CY 2000 dollars. The total cost was fairly equally distributed between medication (37\%), laboratory (36\%), and clinical pharmacist labor costs for monitoring (27\%).

A separate economic analysis study ${ }^{13}$ utilized a medication cost (at average wholesale price) of $\$ 20.40$ (versus $\$ 19.09$ in our study) and a monitoring cost (health care provider and lab) of $\$ 22.61$ to $\$ 33.22$, based on the 1999 Medicare fee schedule (versus $\$ 32.16$ in our study); their assumptions were based on 1 INR test per patient per month, whereas our actual results were an average of 1.1 INR tests per patient per month. The total PPPM (for 1999) in their separate study was calculated to be $\$ 46.81$, 9\% less than our estimated total PPPM cost of $\$ 51.25$ in CY 2000 dollars. Therefore, the total PPPM cost for personnel, laboratory, and drugs for outpatient anticoagulants and monitoring was approximately \$47 in 1999 and \$51 in 2000. A compounded 5\% annual inflation rate in these medical and drug costs in 2000 would yield a conservative total PPPM cost of $\$ 62.30$ in 2004 for oral anticoagulant monitoring of warfarin therapy.

In our study, $19.6 \%$ of the AF patients maintained on warfarin were either at low or moderate risk for stroke; it is unknown how many low-risk AF patients were maintained on aspirin only because they were excluded from the analysis. Go et al. determined that 23\% of all AF patients are at low risk, using ACCP criteria in a managed care population. ${ }^{14}$ Current guidelines ${ }^{5}$ recommend "aspirin only" for low-risk AF patients and either aspirin or warfarin for AF patients with a moderate risk factor for stroke. Patients with more than 1 moderate risk factor (aged 65 to 75 years, diabetes, or coronary artery disease) would be expected to benefit more from warfarin therapy. The benefit versus the risk of long-term warfarin compared with aspirin therapy should be reassessed with the physician in low- to moderaterisk patients, particularly since the annual cost of the anticoagulation service is nearly the same for low-risk and moderate-risk AF patients. The medication, laboratory, and pharmacist monitoring costs would obviously be significantly reduced in a majority of these patients if aspirin monotherapy is indicated.

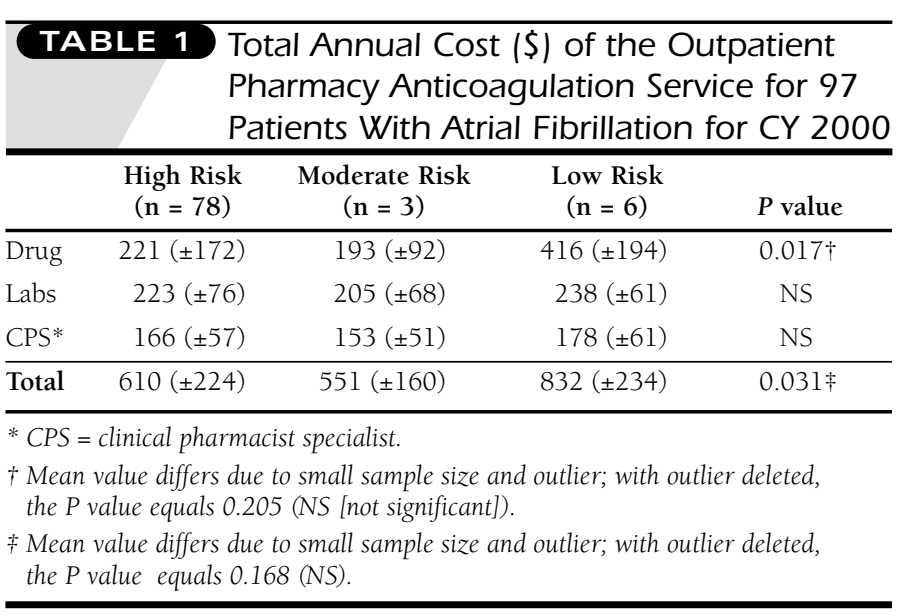

\section{TABLE 2 Per-Patient-Per-Month Cost of the Outpatient Pharmacy Anticoagulation Service for 97 Patients With Atrial Fibrillation for CY 2000}

\begin{tabular}{lll}
\hline Anticoagulant medications + dispensing fee* & 19.09 & $(37 \%)$ \\
\hline INR test + laboratory fee $\dagger$ & 18.38 & $(36 \%)$ \\
\hline Clinical pharmacy specialist $\ddagger$ & 13.78 & $(27 \%)$ \\
\hline Total & 51.25 & \\
\hline
\end{tabular}

* Drug cost is the actual average monthly health plan cost plus dispensing fee.

$\dagger$ INR = international normalized ratio. INR cost is the actual average monthly health plan cost; lab personnel cost and supplies are estimated in laboratory fee. \# Pharmacist cost is monthly cost estimated based on salary, benefits, and number of consults (1,288 per year) for a 24-hour, 7-day-per-week service. Pharmacist cost was determined for monitoring chronic atrial fibrillation patients only.

TABLE 3 Number and Percentage of Monthly Lab Tests Within INR* Goal Versus the Risk for Ischemic Stroke for Atrial Fibrillation

\begin{tabular}{lcccc}
\hline Risk for Stroke & At Goal & Not at Goal & Total No. INRs & $P$ value \\
\hline Low $(\mathrm{n}=6)$ & $42(68.9 \%)$ & $19(31.1 \%)$ & 61 & NS \\
\hline Moderate $(\mathrm{n}=12)$ & $71(57.7 \%)$ & $52(42.3 \%)$ & 123 & NS \\
\hline High $(\mathrm{n}=74)$ & $428(58.6 \%)$ & $302(41.4 \%)$ & 730 & NS \\
\hline Total $(\mathrm{n}=92)$ & $541(59.2 \%)$ & $373(40.8 \%)$ & 914 &
\end{tabular}

* INR = international normalized ratio. NS = not significant.

The quality of warfarin control in OPAS was measured by the time spent in the target INR range rather than an assessment of clinical outcomes. The relationship between anticoagulation control and clinical outcomes, however, is well established. ${ }^{15} \mathrm{In}$ this study, the percentage within the individual patient's stated INR goal was $60.4 \%$ for nonvalvular AF patients. This result 
compares favorably with that found in a similar study in a "bestpractice" physician-directed anticoagulation clinic (54.9\%). ${ }^{12}$ The incidence of INR values above (14.3\%) or below (25.2\%) goal in this study also compared favorably with the physician practice where $18.3 \%$ of values were above goal and $26.7 \%$ below goal.

Cost avoidance was not addressed in this study. Cost avoidance is defined as health care savings from a lower incidence of thromboembolic and hemorrhagic complications resulting from interventions provided by the anticoagulation service. By keeping patients within their INR range more often, the risk of stroke, bleeding, and death would be expected to be lower, resulting in lower health care costs, which could offset the higher monitoring costs for providing the anticoagulation service. Chiquette et al. ${ }^{16}$ compared the costs and efficacy of a dedicated pharmacist directed comprehensive anticoagulation service (not limited to AF patients) with "usual care" in a university setting. In both low (INR 2 to 3) and high intensity (INR 2.5 to 4.5) patients, the pharmacist-directed service had significantly fewer episodes of major bleeding and thromboembolic disease. The cost savings from fewer hospitalizations and ER visits alone were estimated at \$132,086 per 100 patients (in 1998 dollars). Their "cost avoidance" PPPM was calculated to be $\$ 110$ compared with usual care. Wilt et al. ${ }^{17}$ also demonstrated the costeffectiveness of a pharmacy-based anticoagulation service because of improved clinical outcomes.

Campbell et al. ${ }^{13}$ published an economic analysis comparing the costs of AF patients in anticoagulation clinics with usual medical care. In addition to medication, laboratory, and monitoring personnel costs, their data included incidence rates for all adverse reactions (thromboembolic and hemorrhagic events) based on a comprehensive analysis of the medical literature. Their economic model demonstrated that the total costs were reduced by $41 \%$ in the systematic care of an anti-coagulation clinic, primarily due to a significant reduction in the incidence of ischemic strokes. The cost avoidance PPPM (in 1999 dollars) was estimated to be $\$ 89.22$, which more than offset the estimated medication, lab, and monitoring PPPM of $\$ 46.81$.

While an anticoagulation service is generally considered to be more effective than usual care at keeping patients at or near their INR goal, $25 \%$ to $50 \%$ of patients prescribed warfarin continue to be at risk for an ischemic stroke or intracranial bleed. The more specific oral direct thrombin inhibitor ximelagatran is expected to be approved by the FDA for AF in early 2005. This drug has proven to be at least as effective as warfarin with less risk of bleeding. ${ }^{18}$ It will be prescribed in a fixed-dose, twice-daily regimen with minimal drug-drug or drug-diet interactions. Ximelagatran eliminates the need for INR testing, though it may require other laboratory monitoring. This and other future medications may reduce the work of an anticoagulation monitoring service and increase the importance of measuring alternate medical costs necessary to achieve similar clinical and service outcomes.

\section{Limitations}

There are several limitations of our study. It was retrospective in design and comparisons with a control group were not feasible. OPAS was initiated in 1995, and cost data were not available to permit an analysis of costs prior to and after implementation of the service. Coding errors for AF and risk factors for stroke may have occurred but were minimized by chart review and comparison of the data in the clinical database with data in the administrative claims database.

The PPPM cost was derived from a combination of actual health plan costs for drug and laboratory costs and estimated costs for usual and customary fees and pharmacist consults based on the number of INR lab results. The medication costs may be understated if some patients opted to have some refills of their medications dispensed via mail order or at an independent pharmacy. The cost of the drug would also be expected to be higher with the use of brand-name anticoagulants. In order to capture complete anticoagulant monitoring costs in AF patients, this study included the infrequent, but costly, low-molecular-weight heparin (enoxaparin) bridging therapies.

Personnel costs were correlated with the actual number of INR consults since each laboratory result initiated an intervention by the pharmacist. OPAS was decentralized, with multiple CPSs (covering multiple sites) with varying levels of experience and salary; the pharmacists had clinical responsibilities in addition to OPAS. To improve the accuracy of the estimate of personnel costs, pharmacists were independently polled to validate the average time per INR consult (10 to 20 minutes each) and low-molecular-weight heparin bridging (180 minutes each).

A prospective study with a time-motion analysis would be necessary to validate the self-reported time estimates in this study. The cost of the service would be expected to be higher with physician monitoring. Additional cost savings may be possible with a dedicated centralized anticoagulant service. The monitoring and laboratory costs for newly diagnosed AF patients (approximately 30\% of the AF population) were not included in this analysis and would be expected to be higher because of additional interventions and INR lab testing as well as the time necessary for initial patient education.

The relative cost-effectiveness of using clinical pharmacists versus other health care professionals may also be of interest and should be evaluated to compare relative financial efficiencies, preferably in the context of intermediate clinical outcomes (e.g., percentage of time in the target INR range) or ultimate clinical outcomes (e.g., ischemic stroke and bleeding). Reliable analysis of complications such as fatal and nonfatal strokes or bleeding was precluded by the relatively small number of patients, short (1 year) duration of this study, infrequent incidence of stroke, and the study design. Cost-avoidance analysis of additional emergency room visits or hospital admissions precipitated by ineffective maintenance of therapy near INR goal would be likely to overwhelm the actual costs of an oral antico- 
agulant monitoring service.

The effectiveness of OPAS was assessed by indirect measures such as percentage of INR values within or near goal. In our study, $74.6 \%$ of nonvalvular AF patients were "near" their target goal. Guidelines exist for the utilization of less-intensive anticoagulation therapy (INR values between 1.8 and 1.99 were defined as near goal) in elderly patients older than 75 years, ${ }^{19}$ but these guidelines may be subject to review since recent research has demonstrated that the mortality rate in AF patients apparently does not differ with INR values in the range of 1.5 to 1.9 versus INR values $<1.5 .{ }^{6}$ These recent study results strongly support an INR goal of $>2$ in high-risk AF patients of all ages.

\section{Conclusion}

The average PPPM cost of operating an OPAS for AF patients on chronic anticoagulant therapy was determined to be $\$ 51.25$, or an estimated $\$ 62.30$ in CY 2004 dollars. These costs included bridge therapy with low-molecular-weight heparain (enoxaparin) for the $4 \%$ of study patients that required this intervention. The cost for OPAS did not differ significantly in patients with high, moderate, or low risk levels for ischemic stroke. As a measure of the quality of warfarin control in OPAS, nonvalvular AF patients were within their stated INR goal $60.4 \%$ of the time. Since an anticoagulation service can help keep AF patients within their INR goal, health maintenance and other managed care organizations will be interested in determining the actual costs of a service compared with the incremental costs of newer anticoagulation therapies that may require less monitoring and attention of health professionals.

\section{ACKNOWLEDGMENTS}

Administrative, technical, and/or material support was provided by Elizabeth Barham, PharmD; Corrie Crews, PharmD; Becky Hamilton, PharmD; and Douglas Roblin, PhD, all at Kaiser Permanente, Atlanta, Georgia.

\section{DISCLOSURES}

Funding for this research was provided by AstraZeneca LP and was obtained by author Robert J. Anderson, who serves on the Exanta Advisory Board for AstraZeneca LP.

\section{REFERENCES}

1. Ryder KM, Benjamin EJ. Epidemiology and significance of atrial fibrillation. Am J Cardiol. 1999;84:131-38.
2. Feinberg WM, Blackshear JL, Laupacis A, Kronmal R, Hart RG. The prevalence of atrial fibrillation: analysis and implications. Arch Intern Med. 1995; 155:469-73

3. Atrial Fibrillation Investigators. Risk factors for stroke and efficacy of antithrombotic therapy in atrial fibrillation. Analysis of pooled data from five randomized controlled trials. Arch Intern Med. 1994;154(13):1449-57.

4. Wolf PA, Abbott RD, Kannel WB. Atrial fibrillation as an independent risk factor for stroke: the Framingham Study. Stroke. 1991;22:983-88.

5. Albers G, Dalen J, Laupacis A, Manning WJ, Petersen P, Singer DE. Antithrombotic therapy in atrial fibrillation. Chest. 2001;119(suppl):194S-206S.

6. Hylek EM, Go AS, Chang Y, Jensvold NG, Henault LE, et al. Effect of intensity of oral anticoagulation on stroke severity and mortality in atrial fibrillation. New Engl J Med. 2003;349:1019-26.

7. Hylek EM, Singer DE. Risk factors for intracranial hemorrhage in outpatients taking warfarin. Ann Intern Med. 1994;120:897-902.

8. Gullov AL, Koefoed BG, Petersen P. Bleeding during warfarin and aspirin therapy in patients with atrial fibrillation: the AFSAK 2 Study. Arch Intern Med. 1999;159(12):1322-28.

9. Pengo V, Legnani C, Noventa F, Palarenti G. Oral anticoagulant therapy in patients with nonrheumatic atrial fibrillation and risk of bleeding - a multicenter inception cohort study. Thromb Haemost. 2001;85(3):418-22.

10. Go AS, Hylek EM, Chang Y, Phillips KA, Henault LE, et al. Anticoagulation therapy for stroke prevention in atrial fibrillation: how well do randomized trials translate into clinical practice? JAMA. 2003;290:2685-92.

11. Albers GW, Yim J, Josephine M, Belew KM, Bittar N, et al. Status of antithrombotic therapy for patients with atrial fibrillation in university hospitals. Arch Intern Med. 1996;156:2311-16.

12. Samsa GP, Matchar DB, Goldstein LB, Bonito AJ, Lux LJ, et al. Quality of anticoagulation management among patients with atrial fibrillation. Arch Intern Med. 2000;160(7):967-73.

13. Campbell PM, Radensky PW, Denham CR. Economic analysis of systematic anticoagulation management vs. routine medical care for patients on oral warfarin therapy. Dis Manage Clin Outcomes. 2000;2:1-8.

14. Go AS, Hylek EM, Phillips, KA, et al. Implications of stroke risk criteria on anticoagulation decision in non-valvular atrial fibrillation: the Anticoagulation and Risk Factors in Atrial Fibrillation (ATRIA) Study. Circulation. 2000;102:11-13.

15. Hirsh J, Dalen JE, Deykin D, Poller L, Bussey HA. Oral anticoagulants: mechanism of action, clinical effectiveness and optimal therapeutic range. Chest. 1995;108(suppl):231s-246s.

16. Chiquette E, Amato MG, Bussey HI. Comparison of an anticoagulation clinic with usual medical care: anticoagulation control, patient outcomes and health care costs. Arch Intern Med. 1998;158(15):1641-47.

17. Wilt VM, Gums JG, Moore LM. Outcome analysis of a pharmacist-managed anticoagulation service. Pharmacotherapy. 1995;15(6):732-39.

18. Halperin JL. Executive Steering Committee, SPORTIF III, and V Study Investigators. Ximelagatran compared with warfarin for prevention of thromboembolism in patients with nonvalvular atrial fibrillation: rationale, objectives, and design of a pair of clinical studies and baseline patient characteristics (SPORTIF III and V). Am Heart J. 2003;146:431-38.

19. Fuster V, Ryden LE, Asinger RW, et al. ACC/AHA/ESC guidelines for the management of patients with atrial fibrillation. Circulation. 2001;104:2118-50. 